\title{
CT screening for lung cancer in the UK: position statement by UKLS investigators following the NLST report
}

\author{
John Kirkpatrick Field, ${ }^{1}$ David Baldwin, ${ }^{2}$ Kate Brain, ${ }^{3}$ Anand Devaraj, ${ }^{4}$ Tim Eisen, ${ }^{5}$ \\ Stephen W Duffy, ${ }^{6}$ David M Hansell, ${ }^{7}$ Keith Kerr, ${ }^{8}$ Richard Page, ${ }^{9}$ Mahesh Parmar, ${ }^{10}$ \\ David Weller, ${ }^{11}$ Paula Williamson, ${ }^{12}$ David Whynes, ${ }^{13}$ UKLS Team*
}

For numbered affiliations see end of article.

\section{Correspondence to}

Professor John Kirkpatrick Field, Department of Molecular \& Clinical Cancer Medicine, University of Liverpool, 200 London Road, Liverpool L3 9TA, UK; j.k.field@liv.ac.uk

\section{*UKLS Team}

Professor John K Field, (Cl)

University of Liverpool.

Dr David Baldwin, Nottingham, University Hospital.

Dr Kate Brain, Cardiff University. Dr Anand Devaraj, St George's Hospital, London.

Professor Tim Eisen, University of Cambridge.

Professor Stephen Duffy, Barts and The London.

Professor David Hansell, Royal Brompton Hospital.

Professor Keith Kerr, Aberdeen Royal Infirmary.

Mr Richard Page, Liverpool Heart \& Chest Hospital.

Professor Mahesh Parmar

Medical Research Council

Clinical Trial Unit, London.

Professor David Weller,

University of Edinburgh.

Professor Paula Williamson,

University of Liverpool.

Professor David Whynes,

University of Nottingham.

Received 17 April 2011

Accepted 3 June 2011

Published Online First

1 July 2011

\section{ABSTRACT}

Background The imminent publication of a randomised controlled trial from the USA that suggests CT screening reduces mortality from lung cancer by more than $20 \%$, may potentially lead to one of the most important developments in lung cancer care. However, there remain important questions about the applicability of the results to the UK and the clinical effectiveness of this intervention, including its feasibility and cost-

effectiveness.

Objective To describe the remaining questions that need to be answered by further research and to comment on the use of CT screening in the UK outside a clinical trial.

Methods The detailed design process of the UKLS protocol and international discussions were used to identify the research questions that remain to be answered and to inform those who may choose to consider offering CT screening, before these questions are answered.

Results A series of research imperatives have been identified and we advise that CT screening should be part of the ongoing clinical trial in the UK, currently in the pilot phase (UKLS). UKLS is randomising 4,000 individuals for the pilot and a total of 32,000 for the main study.

Conclusion There remain unresolved issues with respect to CT screening for lung cancer. These include its feasibility, psychosocial and cost-effectiveness in the UK, harmonisation of CT acquisition techniques, management of suspicious screening findings, the choice of screening frequency and the selection of an appropriate risk group for the intervention. UKLS is aimed at resolving these issues.

Lung cancer is the most common cause of death from cancer across the world and is responsible for over 33500 deaths per year in the UK. However, if found at an early stage, lung cancer may be cured, and CT screening offers the potential to do this. Randomised clinical trials (RCTs) have been launched internationally to determine whether CT screening reduces mortality from lung cancer and to determine the benefits, harms and cost effectiveness.

There has been a major initiative in the UK to assess CT screening for early lung cancer, facilitated by the recent funding of the pilot RCT, UK Lung Screen (UKLS), by the National Institute for Health
Research Health Technology Assessment. UKLS is based at the Liverpool Cancer Research Centre, University of Liverpool, and is being undertaken in partnership with Liverpool Heart and Chest Hospital, Papworth Hospital, Cambridge, and the Royal Brompton \& Harefield NHS Foundation Trust, London.

The UKLS pilot trial will randomise 4000 individuals with at least a $5 \%$ risk of developing lung cancer over 5 years as predicted by the Liverpool Lung Project Risk Prediction Model. ${ }^{1}$ If the pilot shows that a trial is feasible, a further submission will be made to undertake an RCT, randomising 28000 individuals from seven centres in the UK with the same $5 \% 5$-year risk of lung cancer. The 'Wald Single Screen Design'2 has been chosen for the UKLS RCT. The aim of the main UKLS trial is to establish whether a CT screening can reduce lung cancer deaths by at least 30\% and to determine the cost effectiveness of CT screening in the UK.

In November 2010, the National Lung Screening Trial (NLST) was stopped early in the USA. The director of the National Cancer Institute reported that this randomised trial showed that low-dose CT screening, when compared with screening by chest radiography, resulted in a $20.3 \%$ relative reduction in lung cancer-related mortality and the all-cause mortality was $6.9 \%$ lower in those screened with low-dose CT, relative to those screened with chest radiography. ${ }^{3}$ This trial entered 53000 participants, including both current and former heavy smokers from ages 55 to 74 years. The NLST paper was published in NEJM on $29^{\text {th }}$ June, prior to the IASLC World Conference on Lung Cancer in Amsterdam. ${ }^{4}$ The NLST is the first randomised controlled trial for lung cancer screening to show a significant mortality benefit. The full peer-reviewed publication of NLST results is expected during 2011. There are a number of important differences in the design of NLST and UKLS. Most importantly, NLST employed chest radiographs in the control group whereas UKLS does not; UKLS is a single screen design and it regards smaller nodules that NLST (and indeed any other RCT) evaluated as indeterminate and thus warranting follow-up CT scans.

The UKLS team has concluded that there is an imperative need to proceed with a UK-based randomised trial in order to establish whether 
the results of the NLST trial in the USA can be replicated in the UK.

More specifically, several issues will have to be resolved prior to the implementation of a UK national screening programme, and these include

1. the feasibility and cost effectiveness of CT screening in the UK;

2. defining who is likely to benefit most from CT screening;

3. harmonising the CT acquisition technique, scan reading protocols and acceptable performance levels in the participating centres;

4. determining the value of the individual work-up techniques for suspicious findings and standardisation of performance;

5. determining the optimal surgical management of patients with screen-detected nodules;

6. establishing the optimal screening interval and the number of screening rounds for both screen-negative and screen-positive individuals.

In the light of currently available information from NLST, some individuals may seek to undergo CT screening outside of the UKLS trial. While this is unlikely to be offered by the National Health Service, if and until a cost-effective screening programme is defined by research evidence, screening may be offered by the independent sector. In any non-trial setting, such individuals need to talk with their doctor so that they have access to the best information regarding the potential adverse outcomes and benefits of this approach. This will require a careful assessment of the risk of lung cancer and a full explanation of the likelihood and consequences of finding an indeterminate nodule. The uncertainties outlined above should also be understood by the healthcare professional and patients. The UKLS website will make objective lung cancer screening data widely available to assist such patient/doctor discussions.

UKLS investigators wish to offer the following recommendations to professionals who are considering offering individuals CT screening for lung cancer at this time (outside a clinical trial):

1. Identify the individual's risk of developing lung cancer. CT screening has been shown to reduce mortality only in a specific risk population in the USA.

2. Ensure CT protocols deliver radiation doses at or below those in the latest clinical trials.

3. Ensure that patients are fully informed about the potential harms from the detection of benign abnormalities that require investigation or follow-up.

4. Explain that many more benign abnormalities are found than lung cancers.

5. Ensure that there is relevant expertise in the identification and clinical management of small nodules in the clinical fields of radiology, respiratory medicine, pathology and surgery in the institution undertaking the CT scan.
6. Ensure that clear information on follow-up schedules and the number of repeat CT scans over following years is available and in line with published evidence.

7. Ensure that all patients are informed of warning symptoms of lung cancer after a clear screen, especially if they are at high risk.

The landmark NLST trial has demonstrated that CT is the first clinical tool that demonstrates a significant reduction in lung cancer mortality, in the USA. These initial results support the premise on which the UKLS trial has been developed However, we await the outcome of the UK screening trial to guide National Health Service decision makers on the future of lung cancer screening within the UK.

\section{Author affiliations}

${ }^{1}$ Department of Molecular \& Clinical Cancer Medicine, Institute of Translational Medicine, The University of Liverpool, Liverpool, UK

${ }^{2}$ Respiratory Medicine Unit, David Evans Research Centre, Nottingham University Hospitals, Nottingham, UK

${ }^{3}$ Institute of Medical Genetics, Clinical Epidemiology Interdisciplinary Research Group, Cardiff University School of Medicine, Cardiff, UK

${ }^{4}$ Department of Radiology, St George's Hospital London, London, UK

${ }^{5}$ Cambridge Biomedical Research Centre, Cambridge, UK

${ }^{6}$ Barts and The London School of Medicine and Dentistry, Queen Mary University of London, London, UK

${ }^{7}$ Department of Radiology, Royal Brompton Hospital, London, UK

${ }^{8}$ Department of Pathology, Aberdeen Royal Infirmary, Aberdeen, UK

${ }^{9}$ Department of Surgery, Liverpool Heart \& Chest Hospital, Liverpool, UK

${ }^{10}$ Medical Research Council Clinical Trials Unit, London, UK

${ }^{11}$ Clinical Sciences and Community Health, Division of Community Health Sciences,

University of Edinburgh, Edinburgh, UK

${ }^{12}$ Department of Biostatistics, University of Liverpool, Liverpool, UK

${ }^{13}$ School of Economics, University of Nottingham, Nottingham, UK

Funding The UKLS is funded by the National Institute for Health Research Health Technology Assessment (NIHR HTA).

\section{Competing interests None.}

Contributors JKF, DB, DMH and SWD developed the UKLS position statement, which was amended and then approved by all the remaining members of the UKLS Team ( $K B, A D, T E, K K, R P, M P, D W, P W$ and $D W$ ).

Provenance and peer review Not commissioned; internally peer reviewed.

\section{REFERENCES}

1. Cassidy A, Myles J, van-Tongeren M, et al. The LLP risk model: an individual risk prediction model for lung cancer. Br J Cancer 2008;98:270-6.

2. Baldwin DR, Duffy SW, Wald NJ, et al. UK Lung Screen (UKLS) nodule management protocol: modelling of a single screen randomised controlled trial of low-dose CT screening for lung cancer. Thorax 2011;66:308-13.

3. NLST. http://www.cancer.gov/nlst; http://www.cancer.gov/newscenter/ pressreleases/NLSTresultsRelease.

4. The National Lung Screening Trial Research Team. Reduced Lung-Cancer Mortality with Low-Dose Computed Tomographic Screening. N Engl J Med. 2011 Jun 29. [Epub ahead of print] doi:10.1056/NEJMoa1102873.

\section{Thorax Online Archive}

Visit our Online Archive - available back to 1946. Subscribers may access the entire archive freely. Non-subscribers have free access to all articles prior to 2006. A simple one-time registration is required that grants access to all the free archive content, across all of our specialist titles. To view or to register visit thorax.bmj.com. 